\title{
DERECHO Y POLÍTICAS AMBIENTALES EN LA REGIÓN DE MURCIA
}

\author{
ELISA PÉREZ DE LOS COBOS HERNÁNDEZ \\ Profesora asociada de Derecho Administrativo \\ Universidad de Murcia \\ Abogada \\ SANTIAGo M. Álvarez CARREÑo \\ Profesor titular de Derecho Administrativo \\ Universidad de Murcia
}


Sumario: 1. Breve reflexión introductoria. 2. Manifestaciones de la política y legislación ambiental de la Administración regional en Murcia. 2.1. Mercado de negociación de derechos de emisión de gases de efecto invernadero. 2.2. Lugares de interés comunitario. 2.3. Biodiversidad. 2.4. Plan de Mejora de la Calidad del Aire de la Región de Murcia 2016-2018. 2.5. Premios de Desarrollo Sostenible de la Región de Murcia. 2.6. Plan de Residuos de la Región de Murcia 2015-2020. 2.7. Agricultura. A. Producción ecológica. B. Reglamento de Régimen Interno del Consejo de Agricultura Ecológica de la Región de Murcia. C. Programa de Desarrollo Rural de la Región de Murcia 2014-2020: ayudas para las medidas de agroambiente y clima; ayudas para zonas con limitaciones naturales u otras limitaciones específicas; ayudas para la redotación de comunidades de regantes mediante el aprovechamiento de las aguas residuales regeneradas procedentes de estaciones depuradoras. D. Ayudas para la reconstitución del potencial de producción agrario. E. Ayudas destinadas a los jóvenes agricultores para la creación de empresas agrarias. E. Daños producidos por el granizo y las lluvias torrenciales en el sector agrario. 2.8. Ganadería. 2.9. Pesca. A. Convenio de colaboración para actividades de formación, ordenación y coordinación de la actividad piscícola. B. Fijación de periodos temporales de veda. 2.10. Caza. 3. Ordenanzas locales. 3.1. Murcia: criterios para la graduación en la imposición de sanciones por la comisión de infracciones tipificadas en el artículo 152.1.a) de la Ley 4/2009 de Protección Ambiental Integrada (BORM, núm. 263, de 13 de noviembre de 2015). 3.2. Cehegín: aprobación inicial del "Convenio de encomienda de gestión realizada por la Confederación Hidrográfica del Segura al Ayuntamiento de Cehegín, para el mantenimiento, conservación, vigilancia y gestión diaria de la tubería que parte del canal principal de la Mancomunidad de los Canales del Taibilla hasta la entrada a las instalaciones del embalse del Argos" (BORM, núm. 267, de 18 de noviembre de 2015). 3.3. Blanca. A. Aprobación definitiva de la Ordenanza reguladora de la intervención administrativa en la edificación y uso del suelo (BORM, núm. 269, de 20 de noviembre de 2015). B. Aprobación definitiva del Reglamento regulador del servicio público de abastecimiento de agua potable del municipio de Blanca (BORM, núm. 292, de 19 de diciembre de 2015). 3.4. Bullas: aprobación inicial y aprobación definitiva de la modificación del Reglamento del Consejo Asesor de Medio Ambiente y Planeamiento Urbanístico (BORM, núm. 269, de 20 de noviembre de 2015; BORM, núm. 26, de 2 de febrero de 2016). 3.5. Águilas: anuncio de aprobación inicial y aprobación definitiva de la nueva Ordenanza municipal de uso y disfrute de playas del término municipal de Águilas (BORM, núm. 34, de 11 de febrero de 2016). 3.6. Librilla: aprobación inicial de la Ordenanza municipal de protección de arbolado de interés local (BORM, núm. 60, de 12 de marzo de 2016).

\section{Breve reflexión introductoria}

El período objeto de esta crónica, caro lector, no aporta grandes novedades, apenas algunas resoluciones y convenios. Sin embargo, en el trance de cerrar el relato, conocemos la inquietante aprobación del Decreto-Ley 2/2016, de 20 de abril, de medidas urgentes para la reactivación de la actividad empresarial y del empleo a través de la liberalización y de la supresión de cargas burocráticas. Bajo la apariencia de simples modificaciones orientadas a la inevitable "simplificación administrativa" (eslogan que, por otro lado, de tanto usarlo ha quedado gastado y nadie sabe ya muy bien lo que representa), esta norma lanza misiles desreguladores de gran calado contra la Ley 4/2009, de 14 de mayo, de Protección Ambiental Integrada, por otro lado, inaplicada en su mayor parte durante su tiempo de vigencia (sic). Para adelantar algún contenido relevante que confirme nuestras primeras impresiones, se elimina la “autorización ambiental única”. El Decreto-Ley no introduce, pues, meros retoques. Los autores quedamos reflexionando para poder ofrecerte, querido lector, un análisis más detallado en nuestro próximo encuentro. 


\section{Manifestaciones de la política y legislación ambiental de la Administración regional en Murcia}

2.1. Mercado de negociación de derechos de emisión de gases de efecto invernadero

La Ley $1 / 2005$, de 9 de marzo, por la que se regula el régimen del comercio de derechos de emisión de gases de efecto invernadero, modificada por la Ley 13/2010, de 5 de julio, por la que se transpone la Directiva 2003/87/CE del Parlamento Europeo y del Consejo, de 13 de octubre de 2003, regula el comercio de derechos de emisión de gases de efecto invernadero creado a nivel europeo. De acuerdo con esta legislación, muchas empresas de la Región deben participar en el comercio de derechos de emisión desde el año 2005. Ante el endurecimiento progresivo de las políticas de cambio climático, las empresas y los profesionales deben disponer de información sobre el funcionamiento del mercado de derechos de emisión a fin de definir las estrategias empresariales más adecuadas. Conocer el comportamiento de la oferta y la demanda deviene obligado para aquellas instalaciones con emisiones por encima de los derechos asignados que se plantean "comprar" su déficit, al igual que para las instalaciones menos emisoras que se propongan "vender" su superávit y obtener liquidez por su menor emisión relativa. Esta compraventa de derechos de emisión puede realizarse entre participantes en el mercado de forma bilateral — directamente entre empresas o a través de intermediarios (brokers) — o bien de forma multilateral — acudiendo a las llamadas plataformas de negociación e intermediación de derechos de emisión, también conocidas como mercados electrónicos organizados o bolsas-.

Para facilitar el cumplimiento de las obligaciones que, para las empresas de la Región, se derivan de la Ley 1/2005, de 9 de marzo, la Consejería de Agricultura y Agua, en 2009, y la Consejería de Presidencia, en 2011, firmaron un convenio de colaboración con la mercantil Sistema Europeo de Negociación de $\mathrm{CO}_{2}$, S. L. $\left(\mathrm{SENDECO}_{2}\right)$, con el objetivo de aportar a las empresas y los profesionales de la Región un servicio adicional relacionado con el ámbito financiero del comercio de derechos de emisión ${ }^{1}$. A la vista de los buenos resultados obtenidos, y habiendo expresado $\mathrm{SENDECO}_{2}$ su interés por mantener la colaboración inicial, la Consejería de Agua, Agricultura y Medio Ambiente ha considerado conveniente la puesta en marcha de una nueva edición de esta línea de

\footnotetext{
${ }^{1}$ Sistema Europeo de Negociación de $\mathrm{CO}_{2}$, S. L. $\left(\mathrm{SENDECO}_{2}\right)$, es la única plataforma de negociación e intermediación de derechos de emisión de $\mathrm{CO}_{2}$ existente en España. Se fundó en septiembre de 2004 para proporcionar a las grandes, medianas y pequeñas empresas un sistema basado en internet para la negociación de derechos de emisión de una manera segura, eficiente, transparente e igualitaria.
} 
colaboración para desarrollar actividades que aporten a las empresas y los profesionales de la Región mayores conocimientos sobre el comercio de derechos de emisión y, en especial, sobre su ámbito financiero. Por ello, mediante Resolución de 29 de diciembre de 2015, de la Consejería de Agua, Agricultura y Medio Ambiente, se publica el convenio de colaboración entre la Comunidad Autónoma de la Región de Murcia y $\mathrm{SENDECO}_{2}$ para la divulgación conjunta de las características y funcionamiento del mercado de negociación de derechos de emisión de gases de efecto invernadero ${ }^{2}$.

\subsection{Lugares de interés comunitario}

Mediante la Decisión de Ejecución (UE) 2015/2374, de 26 de noviembre de 2015, la Comisión Europea ha adoptado la novena lista actualizada de lugares de importancia comunitaria de la región biogeográfica mediterránea, que incluye un último ajuste de límites $^{3}$. Los nuevos límites de los LIC figuran en las páginas web del MAGRAMA y de la Región de Murcia — http://www.murcianatural.carm.es/web/guest/56-, en la que pueden descargarse a través del geocatálogo —http:/www.murcianatural.carm.es/ geocatalogo/?q=lic — . Razones de transparencia y seguridad jurídica aconsejan publicar los planos que recogen los límites de los LIC de la Región de Murcia, a excepción de aquellos que han sido declarados ZEC y cuya cartografía ya ha sido publicada en un diario oficial ${ }^{4}$, así como del LIC ES6200048 "Valles submarinos del escarpe de Mazarrón”, cuya declaración como ZEC es competencia del MAGRAMA. De acuerdo con esto, mediante Resolución de 20 de enero de 2016, del Secretario General de la Consejería de Agua, Agricultura y Medio Ambiente, se han hecho públicos los límites de 39 lugares de interés comunitario de la Región de Murcia ${ }^{5}$.

\footnotetext{
${ }^{2}$ Resolución de 29 de diciembre de 2015, de la Consejería de Agua, Agricultura y Medio Ambiente, por la que se publica el convenio de colaboración entre la Comunidad Autónoma de la Región de Murcia y $\mathrm{SENDECO}_{2}$ para la divulgación conjunta de las características y funcionamiento del mercado de negociación de derechos de emisión de gases de efecto invernadero (BORM, núm. 2, de 4 de enero de 2016).

${ }^{3}$ Decisión de Ejecución (UE) 2015/2374, de 26 de noviembre de 2015 (DOUE, L338, de 23 de diciembre).

${ }^{4}$ Decreto núm. 55/2015, de 17 de abril, de Declaración de Zonas Especiales de Conservación y Aprobación del Plan de Gestión Integral de los Espacios Protegidos Red Natura 2000 del Noroeste de la Región de Murcia (BORM, núm. 109, de 14 de mayo de 2015, Suplemento número 1).

${ }^{5}$ Resolución de 20 de enero de 2016, del Secretario General de la Consejería de Agua, Agricultura y Medio Ambiente, por la que se hacen públicos los límites de 39 lugares de interés comunitario de la Región de Murcia (BORM, núm. 17, de 22 de enero de 2016).
} 


\subsection{Biodiversidad}

En su labor de preservación de los valores naturales y paisajísticos de nuestra región, y a fin de conseguir unos sistemas ecológicamente más valiosos y estables frente a las presiones y agresiones externas, la Comunidad Autónoma de la Región de Murcia ha llevado a cabo una planificación y gestión activas que han comportado la inclusión de estos espacios naturales en la red Natura 2000 u otras redes ecológicas de protección o figuras similares. Por su parte, la Fundación "la Caixa" persigue la mejora de la calidad de vida de los ciudadanos mediante un avance social, cultural y ambiental que permita alcanzar un desarrollo humano sostenible. Para ello, desarrolla un gran número de programas medioambientales y sociales dirigidos a la conservación y mejora del entorno natural, así como a la promoción de la integración social de los colectivos de personas desfavorecidas.

A la luz de estos objetivos comunes, la Comunidad Autónoma de la Región de Murcia, a través de la Consejería de Agua, Agricultura y Medio Ambiente, y la Fundación "la Caixa" suscribieron el pasado 28 de diciembre de 2015 un convenio de colaboración para el desarrollo de actuaciones de conservación de la biodiversidad en la Región de Murcia, que se ha hecho público mediante Resolución de 18 de enero de 2016, de la Consejería de Agua, Agricultura y Medio Ambiente ${ }^{6}$. Entre los principales fines del convenio destacan, entre otros, el desarrollo de actuaciones para la conservación, defensa y promoción del medio ambiente en el ámbito territorial de la Comunidad Autónoma de la Región de Murcia, o la actuación estructural sobre los sistemas naturales, de forma que se incida positivamente en los aspectos de la conservación, el uso público y la revitalización socioeconómica.

\subsection{Plan de Mejora de la Calidad del Aire de la Región de Murcia 2016-2018}

La contaminación atmosférica puede definirse como la presencia en la atmósfera de materias, sustancias o formas de energía que impliquen molestia, riesgo o daño para la seguridad o salud de las personas, el medio ambiente y demás bienes de cualquier

\footnotetext{
${ }^{6}$ Resolución de 18 de enero de 2016, por la que se publica el convenio de colaboración entre la Comunidad Autónoma de la Región de Murcia, a través de la Consejería de Agua, Agricultura y Medio Ambiente, y la Fundación bancaria "la Caixa" para el desarrollo de actuaciones de conservación de la biodiversidad en la Región de Murcia (BORM, núm. 17, de 22 de enero de 2016).
} 
naturaleza $^{7}$. La calidad del aire en Europa ha mejorado notablemente en los últimos sesenta años a raíz de los avances científicos, de las exigencias de la sociedad y de la aprobación y aplicación de toda una serie de disposiciones legales. Sin embargo, pese a estos logros, Europa aún no ha conseguido la calidad del aire que prevé su legislación y que desean sus ciudadanos.

A partir de 2014, comienza la implantación de nuevas políticas sobre calidad del aire como respuesta estratégica de la Comisión frente a un problema que puede afectar a los ciudadanos de la UE y al medio ambiente. La Directiva 2008/50/CE del Parlamento Europeo y del Consejo, de 21 de mayo de 2008, relativa a la calidad del aire ambiente y a una atmósfera más limpia en Europa, obliga a las administraciones competentes, en caso de que se superen determinados umbrales, a establecer un plan cuyo objetivo sea mantener y/o reducir los niveles de calidad del aire a unos valores establecidos previamente ${ }^{8}$. Esta directiva se ha traspuesto al ordenamiento jurídico español mediante el Real Decreto 102/2011, de 28 de enero, relativo a la mejora de la calidad del aire, que a su vez desarrolla la Ley 34/2007, de 15 de noviembre, de Calidad del Aire y Protección de la Atmósfera.

Esta última ley otorga al Estado la capacidad para aprobar los planes y programas estatales y así prevenir y disminuir la contaminación atmosférica, siendo el Estado responsable de elaborar y actualizar el inventario nacional de emisiones, dejando a las comunidades autónomas y entes locales la responsabilidad de realizar las mediciones de la calidad del aire y controlar las emisiones a la atmósfera. De acuerdo con esto, mediante Acuerdo de Consejo de Gobierno de 25 de noviembre de 2015, a propuesta de la Consejería de Agua, Agricultura y Medio Ambiente, fue aprobado el Plan de Mejora de la Calidad del Aire de la Región de Murcia 2016-2018, el cual, dada la trascendencia que su implementación ha de tener para las administraciones públicas, los sectores productivos y los ciudadanos, se ha hecho público mediante Resolución de la Dirección General de Calidad y Evaluación Ambiental de 20 de enero de $2016^{9}$.

\footnotetext{
${ }^{7}$ Art. 3 e) de la Ley 34/2007, de 15 de noviembre, de Calidad del Aire y Protección de la Atmósfera.

${ }^{8}$ La Directiva 2008/50/CE del Parlamento Europeo y del Consejo, de 21 de mayo de 2008, relativa a la calidad del aire ambiente y a una atmósfera más limpia en Europa (DOUEL, núm. 152, de 11 de junio de 2008).

${ }^{9}$ Resolución de 20 de enero de 2016, de la Dirección General de Calidad y Evaluación Ambiental, por la que se hace pública la aprobación del Plan de Mejora de la Calidad del Aire de la Región de Murcia 2016-2018 (BORM, núm. 35, de 12 de febrero de 2016). El contenido del Plan de Mejora de la Calidad del Aire de la Región de Murcia 2016-2018 puede consultarse en http://www.carm.es/.
} 


\subsection{Premios de Desarrollo Sostenible de la Región de Murcia}

Los premios en materia de medio ambiente tienen en la Región de Murcia un largo recorrido, convocándose por primera vez en el año 2002 con el nombre de Premios de Calidad Ambiental. En 2006, y recogiendo las recomendaciones contenidas en el Pacto Social por el Medio Ambiente, pasaron a denominarse Premios de Desarrollo Sostenible $^{10}$. La Ley 4/2009, de 14 de mayo, de Protección Ambiental Integrada, ha sido un refuerzo para estos premios dado que, en su título VI, bajo la rúbrica "Fomento del medio ambiente y lucha frente al cambio climático", establece numerosas referencias a la necesidad de impulsar e incentivar desde la Administración regional determinados comportamientos y modelos de desarrollo sostenible. En atención a esto, la Consejería de Agricultura, mediante Orden de 28 de mayo de 2015, convocó la octava edición de los Premios de Desarrollo Sostenible de la Región de Murcia ${ }^{11}$, resuelta por la Consejería de Agua, Agricultura y Medio Ambiente mediante la Orden de 5 de febrero de 2016, por la que se conceden los galardones ${ }^{12}$.

Los premios cumplen un doble objetivo: por una parte, reconocen públicamente el esfuerzo realizado por empresas, instituciones, organizaciones y colectivos de la Región de Murcia en materia de desarrollo sostenible; y, por otra, difunden aquellas experiencias de éxito e ideas que puedan ser referentes para otros. De acuerdo con esto, se integran diferentes modalidades: i) Premios de Desarrollo Sostenible: en los que se valoran los esfuerzos en gestión ambiental sostenible, ecoeficiencia e innovación ambiental, incluyendo, en cuanto a este último aspecto, tecnologías y procedimientos, productos y servicios, y métodos de gestión cuyo impacto sea menos perjudicial para el medio ambiente que otras alternativas disponibles, realizados por empresas y cualquier otro tipo de organización; ii) Premios a la Iniciativa frente al Cambio Climático: en los que se tienen en cuenta las iniciativas, los proyectos y las actividades cuyo objetivo sea

\footnotetext{
${ }^{10}$ Cfr. Protocolo General suscrito entre la Consejería de Industria y Medio Ambiente y las organizaciones representativas de la actividad económica y social de la Región de Murcia para fomentar el desarrollo sostenible en el marco del Pacto Social por el Medio Ambiente, autorizado por el Consejo de Gobierno en sesión de 17 de noviembre de 2006 (BORM, núm. 20, de 25 de enero de 2007).

${ }^{11}$ Orden de 28 de mayo de 2015, de la Consejería de Agricultura, por la que se regula y convoca la octava edición de los Premios de Desarrollo Sostenible de la Región de Murcia (BORM, núm. 125, de 2 de junio de 2015). Vid. Revista Catalana de Dret Ambiental, vol. VI, núm. 2 (2015): 1 - 15 -Crònica-.

${ }^{12}$ Orden de 5 de febrero de 2016, de la Consejería de Agua, Agricultura y Medio Ambiente, por la que se conceden los Premios de Desarrollo Sostenible correspondientes a su octava edición (BORM, núm. 36, de 13 de febrero de 2016).
} 
la mitigación y/o adaptación a los impactos del cambio climático; iii) Premios a la Responsabilidad Social Corporativa en materia de Medio Ambiente: respecto a los proyectos, las actividades y los compromisos asumidos voluntariamente por las empresas y organizaciones empresariales en relación con el medio ambiente en el ámbito de la responsabilidad social corporativa; iv) Premios de Educación Ambiental: en los que se tienen presentes las iniciativas, los proyectos y las actividades que contribuyan a la generación y disposición de información para la educación ambiental y la sensibilización de empresas y ciudadanos; y, por último, v) Premio Mención Especial: que se otorgará tras valorar la trayectoria de ciudadanos, empresas y cualquier tipo de organización especialmente representativos en el campo del medio ambiente.

\subsection{Plan de Residuos de la Región de Murcia 2015-2020}

La aprobación de la Ley 22/2011, de 28 de julio, de Residuos y Suelos Contaminados, además de la necesaria adaptación de la legislación estatal a la Directiva 2008/98/CE, de 19 de noviembre, sobre residuos, determinó la necesaria actualización del régimen jurídico de los residuos, obligando a revisar el proceso de planificación desarrollado hasta el momento en la Región de Murcia. De este modo, una vez examinada la versión preliminar del Plan de Residuos de la Región de Murcia para un nuevo período 20152020 y comprobada su adecuación a la nueva realidad normativa y a la planificación estatal — constituida fundamentalmente por el Plan Nacional Integrado de Residuos para el período 2008-2015-, el Plan de Residuos de la Región de Murcia 2015-2020 se somete a información pública, con lo que se cumple lo dispuesto en el artículo 10 de la Ley 9/2006, de 28 de abril, sobre evaluación de los efectos de determinados planes y programas en el medio ambiente ${ }^{13}$.

\subsection{Agricultura}

A. Producción ecológica

La Consejería de Agua, Agricultura y Medio Ambiente, mediante Resolución de 15 de marzo de 2016, ha aprobado el cuaderno de normas técnicas de la producción ecológica

\footnotetext{
${ }^{13}$ Anuncio por el que se somete a información pública la versión preliminar del Plan de Residuos de la Región de Murcia 2015-2020 (BORM, núm. 258, de 7 de noviembre de 2015).
} 
de la Región de Murcia ${ }^{14}$. Se cumple así lo dispuesto en la Orden de 26 de febrero de 2016, por la que se aprueba el Reglamento de Régimen Interno del Consejo de Agricultura Ecológica de la Región de Murcia. En concreto, el artículo 6 de la referida Orden de 26 de febrero de 2016 dispone que los productos agroalimentarios que vayan a llevar una de las indicaciones que hagan referencia al método ecológico de producción deben obtenerse de acuerdo con las normas establecidas en el título III del Reglamento (CE) 834/2007 del Consejo y en sus disposiciones de aplicación, así como en el cuaderno de normas técnicas del Consejo de Agricultura Ecológica, el cual será aprobado por la Consejería y puesto a disposición de los operadores. Asimismo, en sus artículos 8 y 9 se concreta que las prácticas empleadas en la manipulación, elaboración y/o envasado de los productos ecológicos o en los importados de un país tercero serán las autorizadas en el cuaderno de normas técnicas del Consejo de Agricultura Ecológica. Por lo tanto, la Resolución de 15 de marzo de 2016, por la que se aprueba el cuaderno de normas técnicas de la producción ecológica de la Región de Murcia, deviene esencial para dar cumplimiento a lo anterior.

B. Reglamento de Régimen Interno del Consejo de Agricultura Ecológica de la Región de Murcia

El Consejo de Agricultura Ecológica de la Región de Murcia, creado mediante Orden de 14 de junio de 1999, de la Consejería de Agricultura, Agua y Medio Ambiente, es el órgano encargado de aplicar el sistema de control de la agricultura ecológica en nuestra región. Entre sus funciones destaca la de elaborar el proyecto de Reglamento de Régimen Interno y proponerlo, para su ratificación, a la Consejería de Agua, Agricultura y Medio Ambiente, así como velar por su cumplimiento una vez aprobado $^{15}$. De acuerdo con esto, el Consejo de Agricultura Ecológica ha propuesto a la Consejería de Agua, Agricultura y Medio Ambiente la modificación de diversos artículos de su Reglamento de Régimen Interno, aprobado por Orden de 23 de julio de 2012 de la Consejería de Agricultura y Agua.

\footnotetext{
${ }^{14}$ Resolución de 15 de marzo de 2016, de la Consejería de Agua, Agricultura y Medio Ambiente, por la que se aprueba el cuaderno de normas técnicas de la producción ecológica de la Región de Murcia (BORM, núm. 66, de 21 de marzo de 2016).

${ }^{15}$ Artículo 6. 2. i) de la Orden de 14 de junio de 1999, de la Consejería de Agricultura, Agua y Medio Ambiente, por la que se crea el Consejo de Agricultura Ecológica de la Región de Murcia.
} 
Dado que las modificaciones propuestas afectan a varios de los artículos de la Orden de 23 de julio de 2012, resulta aconsejable su derogación y la publicación íntegra del Reglamento modificado, lo cual se lleva a cabo mediante Orden de 26 de febrero de 2016, de la Consejería de Agua, Agricultura y Medio Ambiente, por la que se aprueba el Reglamento de Régimen Interno del Consejo de Agricultura Ecológica de la Región de Murcia ${ }^{16}$.

C. Programa de Desarrollo Rural de la Región de Murcia 2014-2020: ayudas para las medidas de agroambiente y clima; ayudas para zonas con limitaciones naturales u otras limitaciones específicas; ayudas para la redotación de comunidades de regantes mediante el aprovechamiento de las aguas residuales regeneradas procedentes de estaciones depuradoras

A finales del año 2013 se inicia en la Unión Europea un nuevo período de programación de desarrollo rural 2014-2020 cuyo marco normativo se fundamenta en el Reglamento 1305/2013 del Parlamento Europeo y del Consejo, de 17 de diciembre de 2013, relativo a la ayuda al desarrollo rural a través del Fondo Europeo Agrícola de Desarrollo Rural (FEADER). El Reglamento estructura las distintas medidas de desarrollo rural que pueden cofinanciarse a través del mencionado Fondo, entre las que destacan las destinadas al agroambiente y el clima ${ }^{17}$. Con fecha 3 de julio de 2015 se aprobó el Plan de Desarrollo Rural de la Región de Murcia para el período 2014-2015, en el que se incluyen determinadas medidas con incidencia en la ganadería de la Región de Murcia. Las bases de estas ayudas no se incluyeron en la Orden de 25 de mayo de 2015, de la Consejería de Agricultura y Agua ${ }^{18}$, motivo por el que la Consejería de Agua, Agricultura y Medio Ambiente ha dictado la Orden de 4 de diciembre de 2015, por la

\footnotetext{
${ }^{16}$ Orden de 26 de febrero de 2016, de la Consejería de Agua, Agricultura y Medio Ambiente, por la que se aprueba el Reglamento de Régimen Interno del Consejo de Agricultura Ecológica de la Región de Murcia (BORM, núm. 55, de 7 de marzo de 2016).

${ }^{17}$ Artículo 28 ("Agroambiente y clima") del Reglamento 1305/2013 del Parlamento Europeo y del Consejo, de 17 de diciembre de 2013, relativo a la ayuda al desarrollo rural a través del Fondo Europeo Agrícola de Desarrollo Rural (FEADER).

${ }^{18}$ La Orden de 25 de mayo de 2015, de la Consejería de Agricultura y Agua, por la que se establecen, en la Región de Murcia, las bases reguladoras de determinadas líneas de ayuda correspondientes a la medida 10, "Agroambiente y Clima", y la medida 11, "Agricultura Ecológica", del Programa de Desarrollo Rural de la Región de Murcia 2014-2020, y se aprueba la convocatoria correspondiente al año 2015 de las citadas líneas de ayuda y su posterior modificación realizada mediante la Orden de 5 de agosto de 2015, de la Consejería de Agua, Agricultura y Medio Ambiente. Vid. Revista Catalana de Dret Ambiental, vol. VI, núm. 2 (2015): 1 - 15 -Crònica-.
} 
que se establecen en la Región de Murcia las bases reguladoras de determinadas líneas de ayuda correspondientes a la medida 10, "Agroambiente y clima", relacionadas con actividad ganadera del Programa de Desarrollo Rural de la Región de Murcia 20142020, se aprueba la convocatoria correspondiente al año 2015 de las citadas líneas de ayuda y se modifica la Orden de 25 de mayo de 2015, de la Consejería de Agricultura y Agua.

Asimismo, mediante Orden de 4 de marzo de 2016, la Consejería de Agua, Agricultura y Medio Ambiente establece las bases reguladoras de las líneas de ayuda correspondientes a la medida 13, "Pagos a zonas con limitaciones naturales u otras limitaciones específicas", del Programa de Desarrollo Rural de la Región de Murcia 2014-2020, entre las cuales destacan los pagos compensatorios en zonas de montaña y los pagos compensatorios en zonas con limitaciones naturales ${ }^{19}$.

Por último, mediante esta misma Orden de 4 de marzo de 2016, la Consejería de Agua, Agricultura y Medio Ambiente establece las bases reguladoras de las ayudas para la redotación de comunidades de regantes mediante el aprovechamiento de las aguas residuales regeneradas procedentes de estaciones depuradoras, en el marco del Programa de Desarrollo Rural de la Región de Murcia 2014-2020, operación subvencionable 4.3.2. "Aprovechamiento de aguas residuales para riego"20. Las ayudas reguladas en esta orden tienen como finalidad la consolidación de los regadíos de las comunidades de regantes de la Región de Murcia mediante la incorporación de nuevos caudales de agua para riego procedentes de las estaciones depuradoras de dicho ámbito territorial. Las inversiones se realizarán a través de los proyectos de aprovechamiento de aguas residuales regeneradas presentados por las comunidades de regantes solicitantes.

\section{Ayudas para la reconstitución del potencial de producción agrario}

Las adversidades meteorológicas vividas en el año 2014 motivaron que la Consejería de Agricultura y Agua estableciera, mediante Orden de 30 de diciembre de 2014, las bases

\footnotetext{
${ }^{19}$ Orden de 4 de marzo de 2016, de la Consejería de Agua, Agricultura y Medio Ambiente, por la que se establecen las bases reguladoras de las líneas de ayuda correspondientes a la medida 13, "Pagos a zonas con limitaciones naturales u otras limitaciones específicas" (BORM, núm. 57, de 9 de marzo de 2016).

${ }^{20}$ Orden de 4 de marzo de 2016, de la Consejería de Agua, Agricultura y Medio Ambiente, por la que se establecen las bases reguladoras de las ayudas para la redotación de comunidades de regantes mediante el aprovechamiento de las aguas residuales regeneradas procedentes de estaciones depuradoras, en el marco del Programa de Desarrollo Rural de la Región de Murcia 2014-2020 (BORM, núm. 59, de 11 de marzo de 2016).
} 
reguladoras y la convocatoria de las ayudas para la reconstitución del potencial de producción de almendro, fijando unos períodos concretos para posponer el calendario de reconstitución aprobado. Sin embargo, debido a las condiciones climáticas y a la falta de planta disponible en los viveros, y con el fin de garantizar una gestión eficiente, ha sido necesario modificar el plazo de presentación de las solicitudes de estas ayudas. Para ello, mediante Orden de 5 de noviembre de 2015, de la Consejería de Agua, Agricultura y Medio Ambiente, se ha corregido la Orden de 30 de diciembre de 2014, de la Consejería de Agricultura y Agua, por la que se establecen las bases reguladoras y se convocan ayudas para la reconstitución del potencial de producción agrario como consecuencia de adversidades climáticas producidas en el año hidrológico 2014 en el marco de los programas de desarrollo rural 2007-2013 y 2014-2020 FEADER de la Región de Murcia. En este sentido, se redacta un nuevo apartado 3 del artículo 16 por el que se concreta que únicamente se podrán solicitar modificaciones que pospongan el calendario de reconstitución durante los tres primeros meses de las anualidades 2016 y 2017. No obstante, durante el período comprendido entre el 10 de noviembre y el 10 de diciembre de 2015 se podrán solicitar modificaciones de las concesiones otorgadas con cargo a las anualidades 2016 o siguientes.

E. Ayudas destinadas a los jóvenes agricultores para la creación de empresas agrarias

El Reglamento 1305/2013, relativo a la ayuda al desarrollo rural a través del Fondo Europeo Agrícola de Desarrollo Rural (FEADER), establece un conjunto de medidas destinadas a la inversión en explotaciones agrarias y a la creación de empresas por jóvenes agricultores. En concreto, mediante las ayudas previstas en los artículos 17 y 19 del Reglamento 1305/2013 se pretende fomentar la creación de empleos en las zonas rurales, garantizar la viabilidad de las nuevas actividades y mejorar el rendimiento global y la sostenibilidad de la explotación agraria.

La creación de empresas, y, en concreto, aquellas cuyo objeto sea la actividad agraria y sean acometidas por jóvenes agricultores, resultan de especial interés para la Región de Murcia al estimular no solo el relevo generacional en el sector agrario y la inclusión social, sino también el desarrollo equilibrado, sostenible, económico y territorial de las zonas rurales. Para ello, la Consejería de Agua, Agricultura y Medio Ambiente, mediante Orden de 26 de noviembre de 2015, establece las bases reguladoras de las 
ayudas destinadas a los jóvenes agricultores para la creación de empresas agrarias y la inversión de sus explotaciones en el marco del Programa de Desarrollo Rural de la Región de Murcia 2014-2020, aprobando la convocatoria correspondiente al año 2015 de las citadas líneas de ayuda ${ }^{21}$.

F. Daños producidos por el granizo y lluvias torrenciales en el sector agrario

Las inclemencias climatológicas vividas durante los meses de abril, mayo, julio, agosto y septiembre de 2015 motivaron que desde la Asamblea Regional se instase al Gobierno Regional la instauración de una línea de créditos preferenciales dirigidos a paliar los daños producidos por el granizo y las lluvias torrenciales en el sector agrario. De este modo, mediante Resolución de 28 de diciembre de 2015, la Consejería de Agua, Agricultura y Medio Ambiente ha publicado el texto del Convenio de colaboración entre la Administración general de la Comunidad Autónoma de la Región de Murcia, a través de la Consejería de Agua, Agricultura y Medio Ambiente, y diversas entidades financieras para la formalización de préstamos preferenciales a titulares de explotaciones agrícolas afectadas por temporales de granizo y lluvias torrenciales ${ }^{22}$. El Convenio tiene por objeto establecer los términos y las condiciones de líneas de financiación preferentes para facilitar, potenciar y ampliar el crédito en la Región de Murcia para aquellos agricultores y empresas que se encuentren afectados por los temporales de granizo y lluvias torrenciales acaecidos en los meses de abril, mayo, julio, agosto y septiembre de 2015 .

\subsection{Ganadería}

Dentro de la producción primaria, el sector porcino destaca por su crecimiento y desarrollo. Sin embargo, de forma paralela a su intensificación, este sector acarrea el

\footnotetext{
${ }^{21}$ Orden de 26 de noviembre de 2015, de la Consejería de Agua, Agricultura y Medio Ambiente, por la que se establecen las bases reguladoras de las ayudas destinadas a los jóvenes agricultores para la creación de empresas agrarias y la inversión de sus explotaciones en el marco del Programa de Desarrollo Rural de la Región de Murcia 2014-2020 (BORM, núm. 277, de 30 de noviembre de 2015).

${ }^{22}$ Resolución de 25 de enero de 2016, por la que se publica el documento de adhesión de la entidad financiera Caja de Ahorros y Monte Piedad de Zaragoza, Aragón y Rioja (Ibercaja) al convenio de colaboración entre la Administración general de la Comunidad Autónoma de la Región de Murcia, a través de la Consejería de Agua, Agricultura y Medio Ambiente, y diversas entidades financieras para la formalización de préstamos preferenciales para el anticipo de ayudas directas PAC (BORM, núm. 2, de 4 de enero de 2016).
} 
problema de la producción de deyecciones (purines), vinculadas directamente a este tipo de producciones intensivas. Sin el adecuado tratamiento, los purines pueden convertirse en un gran foco de contaminación medioambiental y provocar fenómenos de eutrofización, contaminación por nitratos y fosfatos, olores, gases de efecto invernadero, etc. Esta particular situación ha llevado a la UE a adoptar en los últimos años una serie de medidas dirigidas a corregir o minimizar el impacto ambiental descrito $^{23}$. El desarrollo de este tipo de políticas, tanto a nivel nacional como comunitario, y el impulso de prácticas agrarias compatibles con el entorno natural son reflejo de una creciente preocupación social por la conservación y estabilidad del medio ambiente.

La legislación específica para la actividad sectorial porcina, constituida por el Real Decreto 324/2000, de 3 de marzo, por el que se establecen normas básicas de ordenación de las explotaciones porcinas, ya recogía una serie de exigencias en materia de utilización de deyecciones y estiércoles, estableciendo los procedimientos para su gestión a fin de ofrecer unas garantías medioambientales adecuadas, sin olvidar que esas deyecciones ganaderas tienen un alto valor biológico dado su contenido en nutrientes agronómicos básicos. Como un elemento de apoyo adicional a dichos procedimientos de gestión y eliminación, el Real Decreto 2818/1998, de 23 de diciembre, sobre producción de energía eléctrica por instalaciones abastecidas por recursos o fuentes de energía renovables, residuos y cogeneración, permite la aplicación de la cogeneración para el tratamiento de purines y su inclusión en el régimen especial eléctrico mediante la instalación de plantas especializadas. Tras diferentes y sucesivos marcos normativos reguladores del régimen especial de la producción eléctrica (el último previsto en la Orden IET/1045/2014, de 16 de junio), los ajustes recientes derivados de la modificación del régimen económico especial del que disfrutaban han conllevado el cierre de las 29 plantas de esta naturaleza existentes en el Estado (tres de ellas en la Región de Murcia), con el evidente efecto negativo que tal situación ha tenido en la producción porcina implicada.

A fin de paliar la situación creada, la Consejería de Agua, Agricultura y Medio Ambiente, mediante Orden de 30 de marzo de 2016, ha aprobado las bases reguladoras de las subvenciones para la gestión y el tratamiento de los purines de aquellas

\footnotetext{
${ }^{23}$ En este sentido, sirva de ejemplo, por todas, la Directiva 91/676/CEE, de 12 de diciembre, relativa a la protección de las aguas contra la contaminación producida por nitratos de origen agrícola.
} 
explotaciones porcinas que se destinaban a las plantas de cogeneración ubicadas en la Región de Murcia, para su secado y transformación, antes de su cierre. La finalidad inicial de estas ayudas es la compensación a los ganaderos con explotaciones porcinas afectadas por dicho cierre, de forma que se posibiliten inversiones que mejoren los planes de gestión de las deyecciones producidas en ellas ${ }^{24}$.

\subsection{Pesca}

A. Convenio de colaboración para actividades de formación, ordenación y coordinación de la actividad piscícola

La Consejería de Agua, Agricultura y Medio Ambiente hizo público, mediante Resolución de 16 de noviembre de 2015, el convenio marco de colaboración existente entre la Comunidad Autónoma de la Región de Murcia, a través de la Consejería de Agua, Agricultura y Medio Ambiente, y la Federación de Pesca de la Región de Murcia, por el que se persigue el desarrollo de actividades de formación, ordenación y coordinación de la actividad piscícola ${ }^{25}$. Entre las cláusulas del convenio destacan, junto con la concreción del plazo de vigencia, las causas de extinción, la financiación, la determinación de las áreas de actuación — como, por ejemplo, la educación y formación en materia medioambiental del pescador deportivo y del personal dedicado a la actividad piscícola - o la creación de una comisión de seguimiento para el control efectivo del convenio.

B. Fijación de periodos temporales de veda

La Consejería de Agua, Agricultura y Medio Ambiente de la Región de Murcia ha venido adoptando, tradicionalmente, medidas de limitación del esfuerzo pesquero de su flota. Para ello, se establecen periodos temporales de veda con el fin de garantizar la

\footnotetext{
${ }^{24}$ Orden de 30 de marzo de 2016, por la que se aprueban las bases reguladoras de las ayudas para la gestión de purines producidos en explotaciones porcinas que se destinaban a plantas de cogeneración antes de su cierre (BORM, núm. 77, de 5 de abril de 2016).

${ }^{25}$ Resolución de 16 de noviembre de 2015, por la que se publica el convenio marco de colaboración entre la Comunidad Autónoma de la Región de Murcia, a través de la Consejería de Agua, Agricultura y Medio Ambiente, y la Federación de Pesca de la Región de Murcia para el desarrollo de actividades de formación, ordenación y coordinación de la actividad piscícola (BORM, núm. 272, de 24 de noviembre de 2015).
} 
plena efectividad del Plan de Gestión Integral para la conservación de los recursos pesqueros en el Mediterráneo afectados por las pesquerías realizadas con redes de cerco, redes de arrastre y artes fijos y menores, para el periodo 2013-2017. Medidas estas cuya continuación, en la actualidad, se ha considerado necesaria ${ }^{26}$. De este modo, mediante Orden de 19 de noviembre de 2015, la Consejería de Agua, Agricultura y Medio Ambiente establece una veda temporal para el ejercicio de la pesca de cerco en aguas interiores de la Región de Murcia durante el periodo comprendido entre los días 6 de diciembre de 2015 y 6 de enero de 2016, ambos inclusive ${ }^{27}$. El ejercicio de la actividad pesquera en dicha modalidad, durante el periodo de veda establecido, será sancionado de conformidad con lo dispuesto en la Ley 2/2007, de 12 de marzo, de Pesca Marítima y Acuicultura de la Región de Murcia.

\subsection{Caza}

Mediante Resolución de 16 de noviembre de 2015, la Consejería de Agua, Agricultura y Medio Ambiente ha hecho público el convenio marco de colaboración entre la Comunidad Autónoma de la Región de Murcia y la Federación de Caza de la Región de Murcia, suscrito el pasado 12 de noviembre de 2015, cuyo objetivo principal se cetra en el desarrollo de actividades de formación, ordenación y coordinación de la actividad cinegética $^{28}$. Entre las cláusulas del convenio destacan, junto con la concreción del plazo de vigencia, las causas de extinción, la financiación, la determinación de las áreas de actuación — como, por ejemplo, la realización de actividades encaminadas a la salvaguarda y el fomento del medio natural en favor de los hábitats y la flora y fauna silvestres, así como de mejora y control de las especies cinegéticas en cotos deportivos - o la creación de una comisión de seguimiento para el control efectivo del convenio.

\footnotetext{
${ }^{26}$ Plan de Gestión Integral para la conservación de los recursos pesqueros en el Mediterráneo afectados por las pesquerías realizadas con redes de cerco, redes de arrastre y artes fijos y menores, para el periodo 2013-2017, aprobado por Orden AAA/2808/2012, de 21 de diciembre.

${ }^{27}$ Orden de la Consejería de Agua, Agricultura y Medio Ambiente, de 19 de noviembre de 2015, por la que se establece una veda temporal para el ejercicio de la pesca de cerco en aguas interiores de la Región de Murcia (BORM, núm. 274, de 26 de noviembre de 2015).

${ }^{28}$ Resolución de la Consejería de Agua, Agricultura y Medio Ambiente, de 16 de noviembre de 2015, por la que se publica el convenio marco de colaboración entre la Comunidad Autónoma de la Región de Murcia, a través de la Consejería de Agua, Agricultura y Medio Ambiente, y la Federación de Caza de la Región de Murcia para el desarrollo de actividades de formación, ordenación y coordinación de la actividad cinegética (BORM, núm. 272, de 24 de noviembre de 2015).
} 


\section{Ordenanzas locales}

3.1. Murcia: criterios para la graduación en la imposición de sanciones por la comisión de infracciones tipificadas en el artículo 152.1.a) de la Ley 4/2009 de Protección Ambiental Integrada (BORM, núm. 263, de 13 de noviembre de 2015).

Por Decreto del teniente de alcalde delegado de Urbanismo, Medio Ambiente y Huerta, de fecha 3 de septiembre de 2015, se ha añadido un nuevo supuesto a los contemplados en el Decreto de 1 de agosto de 2012, del concejal-delegado de Urbanismo y Vivienda $^{29}$, que deberá tenerse en cuenta a la hora de aplicar el artículo 156.2 de la Ley 4/2009, de 14 de mayo, de Protección Ambiental Integrada ${ }^{30}$, como criterio en la graduación de la sanción económica aplicable por la comisión de la infracción administrativa prevista en el artículo 152.1.a) de la Ley 4/2009, de 14 de mayo, de Protección Ambiental Integrada ${ }^{31}$, consistente en la instalación, montaje, ejercicio o explotación, traslado o modificación sustancial de una actividad sin contar con la licencia de actividad, en el caso de actividades sujetas a calificación ambiental.

El nuevo supuesto establece que "cuando habiendo solicitado la licencia de apertura con anterioridad a la constatación de los hechos constitutivos de infracción, se haya obtenido la legalización con fecha anterior a la notificación del decreto de incoación, y siempre que en su funcionamiento no se hayan producido daños al medio ambiente o a las personas, ni se hubiera creado un riesgo objetivo, y por tanto no conste otro tipo de hechos derivados del funcionamiento de la actividad que sean constitutivos de infracción, a excepción de la apertura sin la correspondiente autorización, se impondrá multa de $1.000 € ”$.

\footnotetext{
${ }^{29}$ Anuncio, de 4 de octubre de 2012, del Ayuntamiento de Murcia, de criterios para la graduación en la imposición de sanciones por la comisión de infracciones tipificadas en el artículo 156.2 de la Ley 4/2009 de Protección Ambiental Integrada (BORM, núm. 238, de 13 de octubre de 2012).

30 Artículo 156.2 de la Ley 4/2009, de 14 de mayo, de Protección Ambiental Integrada: "Excepcionalmente, por razón de la escasa o nula trascendencia del hecho sancionado y por resultar claramente desproporcionada la sanción prevista a la vista de las circunstancias concurrentes, podrá aplicarse la sanción establecida para la clase de infracción inmediatamente inferior, debiendo justificarse expresamente y de forma adecuada dicha decisión en el expediente sancionador".

${ }^{31}$ Artículo 152.1.a) de la Ley 4/2009, de 14 de mayo, de Protección Ambiental Integrada: “[...] son infracciones muy graves aplicables a actividades sometidas a licencia de actividad como única autorización ambiental: a) La instalación, montaje, ejercicio o explotación, traslado o modificación sustancial de una actividad sin contar con la licencia de actividad, en el caso de actividades sujetas a calificación ambiental".
} 
Este criterio se aplicará a todos aquellos procedimientos sancionadores que se incoen con posterioridad a la fecha de este decreto, así como a los que se encuentren en trámite.

3.2. Cehegín: aprobación inicial del "Convenio de encomienda de gestión realizada por la Confederación Hidrográfica del Segura al Ayuntamiento de Cehegín, para el mantenimiento, conservación, vigilancia y gestión diaria de la tubería que parte del canal principal de la Mancomunidad de los Canales del Taibilla hasta la entrada a las instalaciones del embalse del Argos" (BORM, núm. 267, de 18 de noviembre de 2015)

Mediante Acuerdo de Pleno, en sesión ordinaria celebrada el día 27 de marzo de 2015, se aprobó inicialmente el contenido del borrador del "Convenio de encomienda de gestión realizada por la Confederación Hidrográfica del Segura al Ayuntamiento de Cehegín, para el mantenimiento, conservación, vigilancia y gestión diaria de la tubería que parte del canal principal de la Mancomunidad de los Canales del Taibilla hasta la entrada a las instalaciones del embalse del Argos". De este modo, el pasado 18 de noviembre se hizo público su clausulado, en el que se fijan, entre otras, cuestiones como el objetivo del convenio, las obligaciones de la Confederación Hidrográfica del Segura, las obligaciones del Ayuntamiento de Cehegín, el régimen de gestión de la tubería, la entrada en vigor y el plazo de vigencia, y las causas de resolución del convenio.

\subsection{Blanca}

A. Aprobación definitiva de la Ordenanza reguladora de la intervención administrativa en la edificación y uso del suelo (BORM, núm. 269, de 20 de noviembre de 2015)

Al no existir reclamaciones en contra, se eleva a definitivo el Acuerdo plenario provisional de 10 de septiembre de 2015 del Ayuntamiento de Blanca, sobre aprobación de la modificación de la Ordenanza reguladora de la intervención administrativa en la edificación y uso del suelo. Dicha ordenanza, estructurada en cuatro títulos, contempla cuestiones como las disposiciones generales referidas a su objeto y ámbito de aplicación y la información turística (título I); la intervención administrativa en el uso del suelo y la edificación (título II); los distintos tipos de procedimientos como, por ejemplo, el de las autorizaciones de obras y otros actos sujetos a licencia; la declaración responsable urbanística; el procedimiento de comunicación previa de obra menor (título III); y el control y la disciplina urbanística (título IV). 
B. Aprobación definitiva del Reglamento regulador del servicio público de abastecimiento de agua potable del municipio de Blanca (BORM, núm. 292, de 19 de diciembre de 2015)

Al no haberse presentado reclamaciones en contra, queda automáticamente elevado a definitivo el Acuerdo plenario provisional de 15 de octubre de 2015 del Ayuntamiento de Blanca sobre aprobación del Reglamento regulador del servicio público de abastecimiento de agua potable del municipio de Blanca. Junto con las disposiciones generales respecto del objeto y el ámbito de aplicación (título I), el Reglamento configura los derechos y las obligaciones de la entidad gestora del servicio y de los usuarios (título II); los elementos materiales del servicio, las instalaciones (título III); los aspectos relacionados con la contratación del suministro (título IV); lectura, facturación y cobro (título V); comprobaciones y defraudaciones (título VI); y régimen sancionador y reclamaciones (títulos VII y VIII).

3.4. Bullas: aprobación inicial y aprobación definitiva de la modificación del Reglamento del Consejo Asesor de Medio Ambiente y Planeamiento Urbanístico (BORM, núm. 269, de 20 de noviembre de 2015; BORM, núm. 26, de 2 de febrero de 2016)

El Pleno del Ayuntamiento de Bullas, en sesión ordinaria celebrada el día 28 de octubre de 2015, acordó la aprobación inicial de la modificación del Reglamento del Consejo Asesor de Medio Ambiente y Planeamiento Urbanístico, sometiéndose el expediente a información pública por el plazo de treinta días, a contar desde el día siguiente a la inserción de este anuncio en el Boletín Oficial de la Región de Murcia ${ }^{32}$. De este modo, vista la inexistencia de reclamaciones en contra, el Acuerdo se ha elevado a definitivo y su texto íntegro se ha publicado el 2 de febrero de $2016^{33}$.

3.5. Águilas: anuncio de aprobación inicial y aprobación definitiva de la nueva

\footnotetext{
${ }^{32}$ Aprobación inicial de la modificación del Reglamento del Consejo Asesor de Medio Ambiente y Planeamiento Urbanístico (BORM, núm. 269, de 20 de noviembre de 2015).

${ }^{33}$ Aprobación definitiva de la modificación del Reglamento del Consejo Asesor de Medio Ambiente y Planeamiento Urbanístico (BORM, núm. 26, de 2 de febrero de 2016).
} 
Ordenanza municipal de uso y disfrute de playas del término municipal de Águilas (BORM, núm. 34, de 11 de febrero de 2016)

El Pleno del Ayuntamiento de Águilas, en sesión ordinaria celebrada el 24 de noviembre de 2015, acordó la aprobación inicial de la nueva Ordenanza municipal de uso y disfrute de playas del término municipal de Águilas. De este modo, vista la inexistencia de reclamaciones o sugerencias en contra, el Pleno del Ayuntamiento de Águilas, en sesión ordinaria de fecha 26 de enero de 2016, aprobó, con carácter definitivo, la redacción final de la nueva Ordenanza municipal de uso y disfrute de playas del término municipal de Águilas, una vez incorporadas a esta parte de las sugerencias aportadas dentro del plazo de información pública. El anuncio se ha publicado en el BORM el 11 de febrero de 2016.

3.6. Librilla: aprobación inicial de la Ordenanza municipal de protección de arbolado de interés local (BORM, núm. 60, de 12 de marzo de 2016)

El Pleno del Ayuntamiento de Librilla, en sesión ordinaria celebrada el día 25 de febrero de 2016, acordó la aprobación inicial de la Ordenanza municipal de protección de arbolado de interés local. Se somete esta a información pública por el plazo de treinta días, pudiendo examinarse el expediente y presentarse las reclamaciones y sugerencias que se estimen oportunas. Si transcurrido dicho plazo no se hubiese presentado ninguna reclamación o sugerencia, el Acuerdo, hasta entonces provisional, se entenderá definitivamente adoptado. 